\title{
Self-consistent description of spin-phonon dynamics in ferromagnets
}

\author{
P. Nieves, ${ }^{1,2}$ D. Serantes, ${ }^{3,4}$ and O. Chubykalo-Fesenko ${ }^{2}$ \\ ${ }^{1}$ ICCRAM, Universidad de Burgos, 09001 Burgos, Spain \\ ${ }^{2}$ Instituto de Ciencia de Materiales de Madrid, CSIC, Cantoblanco, 28049 Madrid, Spain \\ ${ }^{3}$ Instituto de Investigacións Tecnolóxicas and Departamento de Física Aplicada, Universidade de Santiago de Compostela, \\ 15782 Santiago de Compostela, Spain \\ ${ }^{4}$ Department of Physics, University of York, York YO10 5DD, United Kingdom \\ (Received 23 March 2016; revised manuscript received 9 June 2016; published 8 July 2016)
}

\begin{abstract}
Several recently reported exciting phenomena such as spin caloritronics or ultrafast laser-induced spin dynamics involve the action of temperature on spin dynamics. However, the inverse effect of magnetization dynamics on temperature change is very frequently ignored. Based on the density matrix approach, in this work we derive a self-consistent model for describing the magnetization and phonon temperature dynamics in ferromagnets in the framework of the quantum Landau-Lifshitz-Bloch equation. We explore potential applicability of our approach for two cases, inspired by magnetocaloric effect and magnetic fluid hyperthermia. In the first case, the spin-phonon dynamics is governed by the longitudinal relaxation in bulk systems close to the Curie temperature; while in the second case it is described by the transverse relaxation during the hysteresis cycle of individual nanoparticles well below the Curie temperature.
\end{abstract}

DOI: 10.1103/PhysRevB.94.014409

\section{INTRODUCTION}

In the last decade, the number of technological applications involving magnetism and heat has increased. In the data storage industry, for example, new technologies such as heat-assisted magnetic recording (HAMR) [1] and thermally assisted magnetic random access memory (MRAMs) [2] have been developed. Similarly, recently discovered phenomena such as ultrafast all-optically induced magnetization dynamics $[3,4]$ and the spin-Seebeck effect [5] are very appealing from technological perspectives that range from increasing the speed of the magnetization switching to the production of spin-voltage generators. On the other hand, well-known phenomenon such as the magnetocaloric effect [6] has recently been the focus of intensive research due to its possible application for new generation of efficient refrigeration devices near room temperature. Finally, another important effect which uses magnetism and heat is magnetic hyperthermia for cancer treatment [7-9].

Modeling of magnetic and heat dynamics in magnetic materials is a complex task since it involves several different microscopic interactions between spins, electrons, and phonons with many degrees of freedom. Very frequently this is done through the phenomenological rate equations such as the three-temperature or two-temperature models [10]. Recently, Ma et al. [11] developed a self-consistent phenomenological atomistic model for the description of the spin-lattice-electron dynamics which can be applied to simulate ultrafast magnetism experiments, among other thermomagnetic phenomena. The self-consistent spin-lattice-electron dynamics, based on the rate approach, was also introduced by Dvornik et al. [12] for the phenomenological macroscopic Baryakhtar equation for the magnetization dynamics. However, the true quantum mechanical theory for these dynamics is still missing.

At the macroscopic level, the so-called Landau-LifshitzBloch equation [13] (LLB) has been also successfully used to model the magnetization switching dynamics in HAMR [14], domain-wall motion induced by the spin-Seebeck effect [15], and ultrafast magnetism [16-21]. Typically, the LLB models used in these works take into account the dependence of the magnetization dynamics on the bath temperature, however, they do not include the inverse effect, i.e., the change of temperature due to the magnetization relaxation. In this work, we derive a self-consistent model of the spin-phonon dynamics starting from the quantum mechanics and consequently based on the quantum version of the LLB equation [22,23] (qLLB). Our approach includes magnetization dynamics effects on phonon bath temperature. Finally, we give some simple examples of its possible applicability to magnetic refrigeration and magnetic hyperthermia.

In Sec. II, we present the theoretical framework of the spin-phonon dynamics, which we use to obtain the time evolution of the phonon temperature self-consistently with the magnetization dynamics. In Sec. III, we present some results for the model applied to the longitudinal and transverse relaxations of the magnetic system. The concrete examples are inspired by the magnetic refrigeration and magnetic hyperthermia phenomena. In Sec. IV, the conclusions of this work are presented. In order to facilitate the understanding of Sec. II, we included four Appendices. In Appendix A, we introduce the general theory of a spin system interacting with a thermal bath, in which this work is based on, while in Appendix B we show some technical details of the application of this general theory to a simple spin-phonon model used by Garanin in Ref. [22] to derive the qLLB equation. In Appendix C, we proof theoretically the energy conservation of spins and phonons in our model via the first law of thermodynamics. Finally, in Appendix D we also present a self-consistent description of the spin and electron bath coupled dynamics for an electron-"impurity" model.

\section{THEORY}

The self-consistent description of spin-phonon dynamics presented in this work is based on the general theory of a spin 
system interacting with a thermal bath [24]. A summary of this approach is showed in Appendix A. The calculation of the magnetization and temperature bath dynamics from this approach could be a hard task depending on the chosen spinbath model [see Eqs. (A6) and (A7)]. In this work, we consider the same simple spin-phonon model used by Garanin to derive the generalized equation of motion for ferromagnets, the qLLB equation [22].

\section{A. Spin-phonon model}

Initially in this model a magnetic ion is assumed to interact weakly with a thermal phonon bath via direct and the secondorder (Raman) spin-phonon processes with a linear spin operator dependence. Then, the ferromagnetic interactions are taken into account in the mean-field approximation (MFA). The spin-phonon model Hamiltonian reads as

$$
\hat{\mathcal{H}}(t)=\hat{\mathcal{H}}_{s}(t)+\hat{\mathcal{H}}_{p h}+\hat{V}_{s-p h}
$$

where $\hat{\mathcal{H}}_{s}$ describes the spin system energy, $\hat{\mathcal{H}}_{p h}$ describes the phonon energy, $\hat{V}_{s-p h}$ describes the spin-phonon interaction:

$$
\begin{aligned}
\hat{\mathcal{H}}_{s}(t) & =-\hat{\boldsymbol{\mu}} \cdot \mathbf{H}^{\mathrm{MFA}}(t)=\gamma \mathbf{H}^{\mathrm{MFA}}(t) \cdot \hat{\mathbf{S}}, \\
\hat{\mathcal{H}}_{p h} & =\sum_{q} \hbar \omega_{q} \hat{a}_{q}^{\dagger} \hat{a}_{q}, \\
\hat{V}_{s-p h} & =\hat{P}\left(\hat{F}_{d}+\hat{F}_{R}\right),
\end{aligned}
$$

where

$$
\begin{aligned}
\hat{P} & =\eta \cdot \hat{\mathbf{S}}, \\
\hat{F}_{d} & =-\sum_{q} V_{q}\left(\hat{a}_{q}^{\dagger}+\hat{a}_{-q}\right), \\
\hat{F}_{R} & =-\sum_{p, q} V_{p, q} \hat{a}_{p}^{\dagger} \hat{a}_{q} .
\end{aligned}
$$

In the expressions above, $\hat{\boldsymbol{\mu}}=-\gamma \hat{\mathbf{S}}$ is the magnetic moment operator, $\hat{\mathbf{S}}$ is the spin operator, $\gamma=|g| \mu_{B} / \hbar$ is the gyromagnetic ratio where $g$ is the Landé $g$ factor, $\mu_{B}$ is the Bohr magneton, and $\hbar$ is the reduced Planck constant. $\hat{a}_{q}^{\dagger}\left(\hat{a}_{q}\right)$ is the creation (annihilation) operator which creates (annihilates) a phonon with frequency $\omega_{q}$ where $q$ stands for both the wave vector $\mathbf{k}$ and the phonon polarization. The first term in the spin-phonon interaction potential $\hat{V}_{s-p h}$ in Eq. (2) takes into account the direct transformation processes which are characterized by the amplitude $V_{q}$, and the second term describes the Raman processes with amplitudes $V_{p, q}$. The interaction may be anisotropic via the crystal field, which is taken into account through the parameter $\boldsymbol{\eta}$. The vector $\mathbf{H}^{\text {MFA }}$ is an effective field in the MFA given by

$$
\mathbf{H}^{\mathrm{MFA}}(t)=\mathbf{H}_{E}(t)+\mathbf{H}(t)+\mathbf{H}_{K}=\frac{J_{0}}{\mu_{a t}} \mathbf{m}(t)+\mathbf{h}(t),
$$

where $\mathbf{H}_{E}(t)=\left(J_{0} / \mu_{a t}\right) \mathbf{m}(t)$ is the homogeneous part of the exchange field, $J_{0}$ is the exchange parameter related in the MFA to the Curie temperature $T_{c}$ as $J_{0}=3 k_{B} T_{c} S /(S+$ 1), $\mu_{a t}=|g| \mu_{B} S$ is the atomic magnetic moment, $\mathbf{m}(t)=$ $\langle\hat{\boldsymbol{\mu}}(t)\rangle / \mu_{a t}=-\langle\hat{\mathbf{S}}(t)\rangle /(\hbar S)$ is the reduced magnetization, and $\mathbf{h}=\mathbf{H}+\mathbf{H}_{K}$, where $\mathbf{H}$ is the external magnetic field and $\mathbf{H}_{K}$ represents the anisotropy field.

\section{B. Magnetization dynamics}

The calculation of the spin dynamics is somewhat cumbersome. First, from the general theory of a spin system interacting weakly with a thermal bath, one finds that the time derivative of the spin operator expectation value is given by Eq. (A6). From this equation, for the spin-phonon model described by Eq. (1) and using Markov and secular approximations, one arrives to (see Appendix B) [22]

$$
\begin{aligned}
\frac{d \mathbf{m}}{d t}= & -\gamma \mathbf{m} \times \mathbf{h}-K_{2} \frac{\tanh \left(\frac{y_{0}}{2}\right)}{\tanh \left(\frac{y}{2}\right)}\left(\frac{2(S+1) \tanh \left(\frac{y}{2}\right)}{m}-1\right) \\
& \times \frac{\mathbf{m} \times(\mathbf{m} \times \mathbf{h})}{m H^{\mathrm{MFA}}} \\
& -2 K_{2}\left(1-\frac{\tanh \left(\frac{y_{0}}{2}\right)}{\tanh \left(\frac{y}{2}\right)} \frac{\mathbf{m} \cdot \mathbf{H}^{\mathrm{MFA}}}{m H^{\mathrm{MFA}}}\right) \mathbf{m}+\left(K_{2}-K_{1}\right) \\
& \times\left[\frac{(\mathbf{m} \times \mathbf{h})^{2}}{\left(m H^{\mathrm{MFA}}\right)^{2}} \mathbf{m}+\frac{\left(\mathbf{m} \cdot \mathbf{H}^{\mathrm{MFA}}\right) \mathbf{m} \times(\mathbf{m} \times \mathbf{h})}{\left(m H^{\mathrm{MFA}}\right)^{2}}\right],
\end{aligned}
$$

where $y$ is defined through the relation (B11), $y_{0}$ is the equilibrium value of $y$, and $K_{1}$ and $K_{2}$ are given by Eqs. (B8) and (B9), respectively. In stationary dynamic processes $(\mid y-$ $\left.y_{0} \mid \ll y\right)$ and assuming a strong exchange field $\left(H_{E} \gg h\right)$, one arrives from Eq. (4) to the so-called qLLB equation [22,23]

$$
\frac{d \mathbf{m}}{d t}=-\gamma \mathbf{m} \times \mathbf{H}_{\mathrm{eff}}+\gamma \alpha_{\|} \frac{\mathbf{m} \cdot \mathbf{H}_{\mathrm{eff}}}{m^{2}} \mathbf{m}-\gamma \alpha_{\perp} \frac{\mathbf{m} \times\left(\mathbf{m} \times \mathbf{H}_{\mathrm{eff}}\right)}{m^{2}},
$$

where $\mathbf{H}_{\mathrm{eff}}$ is the effective field given by

$$
\mathbf{H}_{\mathrm{eff}}= \begin{cases}\frac{1}{2 \widetilde{\chi}_{\|}}\left(1-\frac{m^{2}}{m_{e}^{2}}\right) \mathbf{m}+\mathbf{h}, & T_{p h}<T_{c} \\ \frac{J_{0}}{\mu_{\mathrm{at}}}\left(\epsilon-\frac{3 m^{2}}{5 A_{s}}\right) \mathbf{m}+\mathbf{h}, & |\epsilon| \ll 1\end{cases}
$$

where $\epsilon=\left(T_{c}-T_{p h}\right) / T_{c}, A_{s}=2(S+1)^{2} /\left([S+1]^{2}+S^{2}\right)$, and $m_{e}=B_{S}\left(\beta J_{0} m_{e}\right)$ is the equilibrium magnetization for $h=0$. The longitudinal susceptibility $\tilde{\chi}_{\|}$can be evaluated in the MFA at $T_{p h}<T_{c}$ as $\tilde{\chi}_{\|}=\mu_{a t} \beta B_{S}^{\prime} /\left(1-\beta B_{S}^{\prime} J_{0}\right)$ where $B_{S}^{\prime}(x)=d B_{S} / d x$ is evaluated at the equilibrium $B_{S}^{\prime}=$ $B_{S}^{\prime}\left(\beta J_{0} m_{e}\right)$. The parameters $\alpha_{\|}$and $\alpha_{\perp}$ in Eq. (5) are the so-called longitudinal and transverse damping parameters, respectively, and they are given by

$$
\begin{gathered}
\alpha_{\|}= \begin{cases}\lambda \frac{2 T_{p h}}{3 T_{c}} \frac{2 q_{s}}{\sinh \left(2 q_{s}\right)}, & T_{p h} \lesssim T_{c} \\
\lambda \frac{2 T_{p h}}{3 T_{c}}, & T_{p h}>T_{c}\end{cases} \\
\alpha_{\perp}= \begin{cases}\lambda\left[\frac{\tanh \left(q_{s}\right)}{q_{s}}-\frac{2 T_{p h}}{3 T_{c}}\left(1-\frac{K_{1}}{2 K_{2}}\right)\right], & T_{p h} \lesssim T_{c} \\
\lambda \frac{2 T_{p h}}{3 T_{c}}, & T_{p h}>T_{c}\end{cases}
\end{gathered}
$$

where $q_{s}=3 T_{c} m_{e} /\left[2(S+1) T_{p h}\right]$ and

$$
\lambda=K_{2} \frac{(S+1)}{S} \frac{\mu_{a t}}{\gamma k_{B} T_{p h}}
$$

is the coupling to the bath parameter. In the isotropic case $\left(\eta_{x}=\eta_{y}=\eta_{z}\right)$, the analysis shows that at high temperatures $K_{1} \simeq K_{2}$ while at low temperatures $K_{1} \ll K_{2}[22,23,25]$. In the simulations presented in this work, we always consider the high-temperature case $\left(K_{1}=K_{2}\right)$. Finally, for modeling purposes $\lambda$ is usually considered as a phenomenological "coupling-to-the bath" parameter. 
Note that the longitudinal damping is related to the longitudinal magnetization relaxation and which has shown good agreement in laser-induced ultrafast magnetization dynamics experiments in materials as $\mathrm{Ni}$ [16], Gd [17], and FePt [21]. The transverse damping is basically related to the relaxation of the ferromagnetic resonance (FMR) mode. Consequently, the transverse damping parameter increases with temperature, consistent with atomistic modeling results (Ref. [26]) and well-known FMR experiments [27,28].

The qLLB equation (5) is very similar to its classical counterpart derived in Ref. [13] using nonequilibrium thermodynamics (the Fokker-Planck equation for a collection of atomic spins described by the LLG equation with phenomenological coupling-to-the bath parameter). Unlike its classical counterpart, qLLB depends on the quantum number $S$ which strongly affects both longitudinal and transverse relaxation time scales. Moreover, due to its quantum mechanical origin, it allows to analyze the magnetization dynamics as function of different microscopic models and temperature dependence of the coupling-to-the bath parameter. For more detailed investigation of this matter, see Ref. [23]. Finally, the quantum spin $S$ could be also used phenomenologically as an adjustable parameter to get a better description of the temperaturedependent magnetization and therefore magnetization dynamics. For example, the transition metals are known to be well described with the Brilloun function with $S=\frac{1}{2}$.

\section{Phonon temperature dynamics}

The general theory of a spin system interacting weakly with a thermal bath also allows to analyze the bath dynamics due to the interaction of phonons with the spin system only, that is, in adiabatic conditions one finds that the time derivative of the bath energy expectation value is given by Eq. (A7). For the spin-phonon model described by Eq. (1), the integrals in Eq. (A7) are calculated following the same procedure as in Eqs. (B1) and (B2). As a result, from Eq. (A7) one arrives to

$$
\begin{aligned}
\frac{d}{d t}\left\langle\hat{\mathcal{H}}_{p h}\right\rangle= & \frac{W_{2} \gamma H^{\mathrm{MFA}}(t)}{2 \hbar}\left\langle\left(\eta^{-} \hat{\boldsymbol{S}}^{+}-e^{-y_{0}} \eta^{+} \hat{\boldsymbol{S}}^{-}\right) \boldsymbol{\eta} \cdot \hat{\mathbf{S}}\right\rangle \\
& +\frac{W_{2} \gamma H^{\mathrm{MFA}}(t)}{2 \hbar}\left\langle\boldsymbol{\eta} \cdot \hat{\mathbf{S}}\left(\eta^{+} \hat{\boldsymbol{S}}^{-}-e^{-y_{0}} \eta^{-} \hat{\boldsymbol{S}}^{+}\right)\right\rangle .
\end{aligned}
$$

On the other hand, from Eq. (B5) one finds

$$
\begin{aligned}
\frac{d}{d t}\left\langle\hat{S}_{z}\right\rangle=- & \frac{W_{2}}{2 \hbar}\left\langle\left(\eta^{-} \hat{S}^{+}-e^{-y_{0}} \eta^{+} \hat{S}^{-}\right) \boldsymbol{\eta} \cdot \hat{\mathbf{S}}\right\rangle \\
& -\frac{W_{2}}{2 \hbar}\left\langle\boldsymbol{\eta} \cdot \hat{\mathbf{S}}\left(\eta^{+} \hat{S}^{-}-e^{-y_{0}} \eta^{-} \hat{S}^{+}\right)\right\rangle .
\end{aligned}
$$

Therefore, the substitution of Eq. (11) into (10) leads to

$$
\frac{d}{d t}\left\langle\hat{\mathcal{H}}_{p h}\right\rangle=-\gamma H^{\mathrm{MFA}}(t) \frac{d}{d t}\left\langle\hat{S}_{z}\right\rangle .
$$

As it was done previously, in order to avoid tedious calculations it was assumed that $\mathbf{H}^{\mathrm{MFA}}(t)=H^{\mathrm{MFA}}(t) \mathbf{e}_{z}$, hence, for the general case Eq. (12) reads as

$$
\frac{d}{d t}\left\langle\hat{\mathcal{H}}_{p h}\right\rangle=-\gamma \mathbf{H}^{\mathrm{MFA}}(t) \cdot \frac{d}{d t}\langle\hat{\mathbf{S}}\rangle=\mu_{a t} \mathbf{H}^{\mathrm{MFA}} \cdot \frac{d \mathbf{m}}{d t},
$$

where the time derivative of the magnetization is given by Eq. (4). Therefore, for $n$ atoms in a unit-cell volume $v_{0}$ the time derivative of the bath temperature is

$$
\frac{d T_{p h}}{d t}=\frac{n}{C_{p h} v_{0}} \frac{d}{d t}\left\langle\hat{\mathcal{H}}_{b}\right\rangle=\frac{M_{s}}{C_{p h}} \mathbf{H}^{\mathrm{MFA}} \cdot \frac{d \mathbf{m}}{d t},
$$

where $C_{p h}$ is the phonon bath specific heat in units of $J K^{-1} m^{-3}$ and $M_{s}=n \mu_{a t} / v_{0}$ is the saturation magnetization at $T=0 \mathrm{~K}$. Equation (14) is related to energy conservation between spins and phonons during the dynamics since energy is conserved in each spin-phonon scattering event at the atomic level [see the quantity $W_{2}$, Eq. (B4)]. In Appendix C, we prove the energy conservation of this model using the first law of thermodynamics. Finally, when Eq. (4) is multiplied by $\mathbf{H}^{\mathrm{MFA}}$, the last two terms in the right-hand side of Eq. (4) cancel each other. Therefore, in the case of stationary dynamic processes $\left(\left|y-y_{0}\right| \ll y\right)$ and a strong exchange field $\left(H_{E} \gg h\right)$, Eq. (14) can be written as

$\frac{d T_{p h}}{d t}=\frac{\gamma \alpha_{\|} M_{s} J_{0}}{C_{p h} \mu_{a t}} \mathbf{m} \cdot \mathbf{H}_{\mathrm{eff}}+\frac{\gamma \alpha_{\perp}^{\prime} M_{s}}{C_{p h}} \frac{(\mathbf{m} \times \mathbf{h})^{2}}{m^{2}} \equiv f\left(\mathbf{m}, T_{p h}\right)$,

where $\alpha_{\perp}^{\prime} \equiv \alpha_{\perp}\left(K_{1}=K_{2}\right)$ is given by Eq. (8) with $K_{1}=K_{2}$. The function $f$ describes how the bath temperature changes per unit time due to the interaction of the bath with the spins. We see that the magnetic transverse relaxation [second term in Eq. (15)] always increases the phonon temperature. On the other hand, the longitudinal relaxation [first term in Eq. (15)] increases (decreases) the bath temperature when $\mathbf{m} \cdot \mathbf{H}_{\text {eff }}>0$ $\left(\mathbf{m} \cdot \mathbf{H}_{\text {eff }}<0\right)$, as it should be. More precisely, since we have

$$
\mathbf{m} \cdot \mathbf{H}_{\mathrm{eff}} \simeq \begin{cases}\frac{1}{2 \widetilde{\chi}_{\|}}\left[m_{e}\left(h_{\|}\right)^{2}-m^{2}\right]+\mathcal{O}\left(\delta m^{2}\right), & T_{p h}<T_{c} \\ \frac{3 J_{0} m}{5 A_{s} \mu_{\mathrm{at}}}\left[m_{e}\left(h_{\|}\right)^{3}-m^{3}\right]+\mathcal{O}(\epsilon), & |\epsilon| \ll 1\end{cases}
$$

where $\delta m=m-m_{e}, m_{e}\left(h_{\|}\right)$is the magnitude of the in-field equilibrium magnetization and $h_{\|}$is the component of field $\mathbf{h}$ along $\mathbf{m}$, then in situations where $m<m_{e}\left(h_{\|}\right)\left[m>m_{e}\left(h_{\|}\right)\right]$ the longitudinal relaxation increases (decreases) the bath temperature.

Note also that it follows from Eq. (14) that the characteristic time scale of the temperature dynamics follows that of the magnetization dynamics. The latter is known to have two characteristic time scales [26,29] for the longitudinal dynamics $\left(\tau_{\|}\right)$and for the transverse one $\left(\tau_{\perp}\right)$ defined by

$$
\tau_{\|}=\frac{\tilde{\chi}_{\|}}{\gamma \alpha_{\|}}, \quad \tau_{\perp}=\frac{m_{e}}{2 \gamma h \alpha_{\perp}},
$$

where $m_{e}$ is the equilibrium value of $m$ at given temperature. The two relaxation characteristic times have different time scales: the longitudinal relaxation occurs in the time scale of smaller than $1 \mathrm{ps}$, while the transverse one in the time scale of $100 \mathrm{ps}-1 \mathrm{~ns}$.

Similarly, in Appendix D we present a self-consistent description of the coupled dynamics between spins and electron bath using an electron-impurity model. The time derivative of the electron bath temperature given by Eq. (D9) may be used in the modeling of laser-induced ultrafast magnetization 
dynamics based on qLLB equation coupled to the twotemperature model for taking into account the specific heat of spins. However, such analysis is beyond the scope of this work.

\section{MODELING RESULTS}

In order to illustrate the potential use of the obtained results, in this section we present some examples of the use of the self-consistent spin-phonon dynamical model derived above. Two cases are examined, for which either the simple magnetic longitudinal relaxation process or the transverse one are the key mechanism for the related magnetothermic effects. The chosen examples correspond to research fields that have been very active during the last decades based on their foreseen relevance for societal needs, but that despite the efforts devoted have not yet achieved their anticipated significance. Thus, the first example, focused on the longitudinal relaxation, deals with magnetic refrigeration, the cooling technology that is environmentally friendly and has high-energy efficiency [30]. The second example, focused on the transverse relaxation, deals with magnetic hyperthermia, a promising treatment for cancer treatment that uses the heat released by magnetic nanoparticles under an ac field to treat the tumor. Advantages of such treatment include the avoidance of the harmful secondary effect of chemotherapies/radiotherapies, local application, and the possibility to treat tumor types that are essentially insensitive to usual chemotherapies and radiotherapies.

It is important to keep in mind that the purpose of these examples is not to undertake any unsolved aspect of the related research fields, but only to illustrate the suitability of the presented model to contribute to their further development. Thus, it must be noted, for example, that we use a simplified description where the magnetization dynamics is described in terms of one qLLB equation. For transverse relaxation, this corresponds to the consideration of a small nanoparticle where the magnetization reversal does not include the formation of nonhomogeneous structures. For the longitudinal relaxation, our approach is suitable for modeling situations close to the Curie temperature, first, because the longitudinal relaxation becomes appreciable in this case and, second, because the most favorable thermodynamical processes there correspond to the change of the magnetization magnitude rather than the domain-wall formation.

\section{A. Magnetic longitudinal dynamics}

First, we focus on the coupled magnetic and phonon temperature dynamics induced by a pure magnetic longitudinal relaxation, that is, processes where the magnetization vector only changes its magnitude with time. One interesting technological application of the coupled magnetic and phonon dynamics is magnetic refrigeration (MR), the cooling technology that makes use of the magnetic longitudinal relaxation in order to change the temperature of a magnetic material [31,32]. MR involves the conversion of spin degrees of freedom to temperature, including both phonon and electron contributions, and shows its higher values at magnetic phase transitions where the longitudinal relaxation process dominates. The potential use of MR as an alternative to conventional cooling techniques has not yet been achieved, thus pointing out the importance
TABLE I. Parameters of $\mathrm{Gd}$ and $\mathrm{Fe}_{3} \mathrm{O}_{4}$ used in the simulations. For $\mathrm{Fe}_{3} \mathrm{O}_{4}$, we have $\widetilde{K}_{1}=\widetilde{K}_{1}^{(c)}$.

\begin{tabular}{lcc}
\hline \hline & $\mathrm{Gd}$ & $\mathrm{Fe}_{3} \mathrm{O}_{4}$ \\
\hline$\mu_{a t}\left(\mu_{B}\right)$ & 7.63 & 4.00 \\
$M_{s}(A / m)$ & $2.14 \times 10^{6}$ & $4.5 \times 10^{5}$ \\
$\widetilde{K}_{1}\left(\mathrm{~J} / \mathrm{m}^{3}\right)$ & $1.3 \times 10^{4}$ & $-1.2 \times 10^{2}$ \\
$\widetilde{K}_{2}^{(c)}\left(\mathrm{J} / \mathrm{m}^{3}\right)$ & & $2.8 \times 10^{2}$ \\
$T_{c}(\mathrm{~K})$ & 293 & 860 \\
$S$ & $7 / 2$ & $5 / 2$ \\
$\lambda$ & 0.001 & 0.05 \\
$\gamma\left(\mathrm{T}^{-1} \mathrm{~s}^{-1}\right)$ & $1.76 \times 10^{11}$ & $1.76 \times 10^{11}$ \\
$C_{p h}\left(\mathrm{Jm}^{-3} \mathrm{~K}^{-1}\right)$ & $1.86 \times 10^{6}$ & $3.48 \times 10^{6}$ \\
\hline \hline
\end{tabular}

of developing theoretical tools able to help on the design of commercially suitable MR-based cooling devices. Next, we will show how the reported model described above can contribute to the modeling, and hence guidance, of the MR research.

For simplicity, we take parameters of Gd, often considered as a modeling material for magnetic refrigeration since $T_{c}$ is closed to room temperature. We take into account the uniaxial anisotropy field $\mathbf{H}_{K}=\left(2 \widetilde{K}_{1} m_{z} / M_{s}\right) \mathbf{e}_{z}$, where $\widetilde{K}_{1}$ is the anisotropy constant, and the external field along the $z$ axis $\mathbf{H}=H_{z} \mathbf{e}_{z}$, so that $\mathbf{h}=\mathbf{H}_{K}+\mathbf{H}$. The parameters of Gd used in the simulations are given by Table I.

In Fig. 1, we show the adiabatic temperature change versus the initial temperature $T_{0}$ in $\mathrm{Gd}$ due to the application of an external magnetic field $B_{z}=\mu_{0} H_{z}=5 \mathrm{~T}\left(\mu_{0}\right.$ is the permeability of free space) along the the magnetization vector $\mathbf{m}=m_{z} \mathbf{e}_{z}$, calculated using the qLLB equation [Eq. (5)] coupled to Eq. (15) (solid line). The results are compared with the experimental data from Ref. [33] (solid dots), showing a very good agreement. Here, we calculated only the temperature change at $T_{p h}<T_{c}$, where the material is ferromagnetic at the initial state. At $T_{p h}>T_{c}$, the initial state is paramagnetic, so that the temperature change may also be calculated using

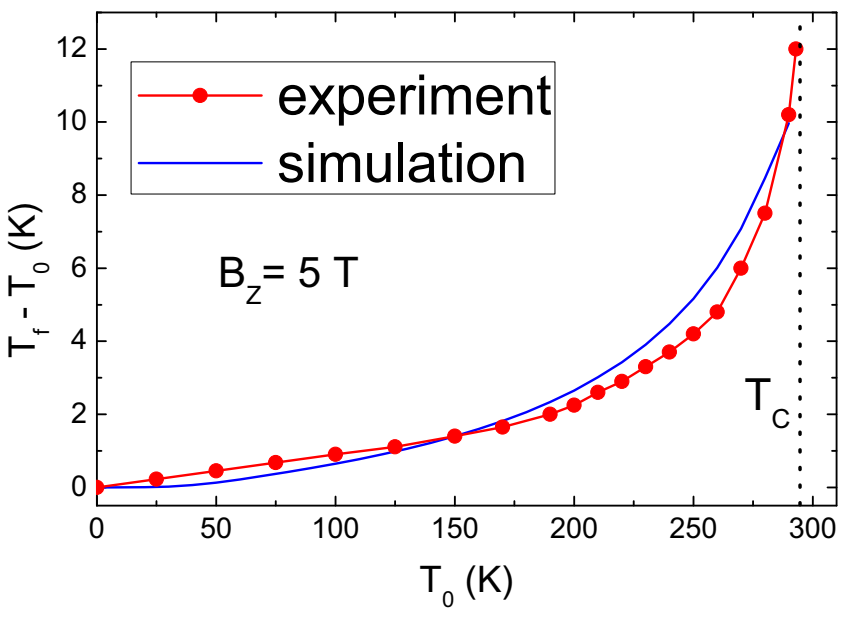

FIG. 1. Temperature change $\Delta T=T_{f}-T_{0}$ versus the initial temperature $T_{0}$ in Gd due to the application of an external magnetic field $B_{z}=\mu_{0} H_{z}=5 \mathrm{~T}$ calculated using the qLLB equation [Eq. (5)] coupled to Eq. (15) (solid blue line) The dotted red line corresponds to the experiment from Ref. [33] (solid dots). 


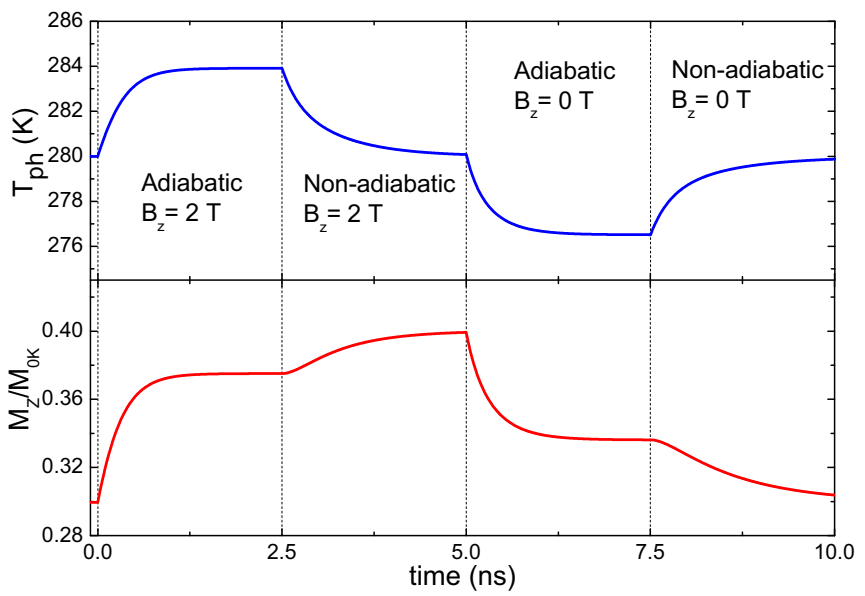

FIG. 2. Refrigeration cycle of Gd obtained by the integration of the qLLB equation (5) coupled to Eq. (15) in the adiabatic processes and to Eq. (18) in the nonadiabatic processes.

the paramagnetic qLLB equation [22], which is obtained by the replacement $\mathbf{H}^{\mathrm{MFA}} \rightarrow \mathbf{H}$ in the qLLB equation given by Eq. (4). We see that the temperature change obtained by simulations is very similar to the experiments.

A simple example of the refrigeration cycle could consist of the following steps: (i) adiabatic process where an external magnetic field is applied, (ii) nonadiabatic process keeping the external magnetic field on, (iii) adiabatic demagnetization removing the external field, and (iv) nonadiabatic process in the absence of the external field. In order to show the applicability of the self-consistent spin-phonon model to magnetic refrigeration, we are going to simulate this refrigeration cycle of Gd. For the nonadiabatic processes, we add Newton's law of cooling in Eq. (15), that is, we coupled the qLLB equation to

$$
\frac{d T_{p h}}{d t}=f\left(\mathbf{m}, T_{p h}\right)-\frac{T_{p h}-T_{\text {room }}}{\tau_{\text {th }}},
$$

where function $f$ is given by Eq. (15), $T_{\text {room }}=280 \mathrm{~K}$, and $\tau_{\text {th }}=400 \mathrm{ps}$. Notice that the dynamics in the nonadiabatic processes are very sensitive to the diffusion model and the value of $\tau_{\mathrm{th}}$. In the present case, we have chosen them arbitrarily, but they need to be adjusted to every specific situation of interest.

In Fig. 2, we show the refrigeration cycle of Gd calculated by the integration of the qLLB equation [Eq. (5)] coupled to the phonon bath through Eq. (15) in the adiabatic and to Eq. (18) in nonadiabatic processes.

As we have shown in Fig. 1, the magnetocaloric effect is more efficient at temperatures close to $T_{c}$. However, as we show in Fig. 3 at temperatures close to $T_{c}$ the magnetization and temperature take more time to reach their equilibrium values. This is due to the phenomenon called critical slowing down which is related to divergence of the longitudinal susceptibility at $T=T_{c}, \tilde{\chi}_{\|} \propto 1 /\left(T_{c}-T\right)$ [23]. In particular, the analysis of the qLLB equation shows that at $T=T_{c}$ the magnetization longitudinal relaxation time follows the expression [23]

$$
\tau_{\|}\left(h, T_{c}\right)=\frac{5 A_{s} \mu_{a t}}{6 \gamma \lambda J_{0} m_{h}^{2}}, \quad m_{h}=\left(\frac{5 A_{s} \mu_{a t} h}{3 J_{0}}\right)^{1 / 3},
$$

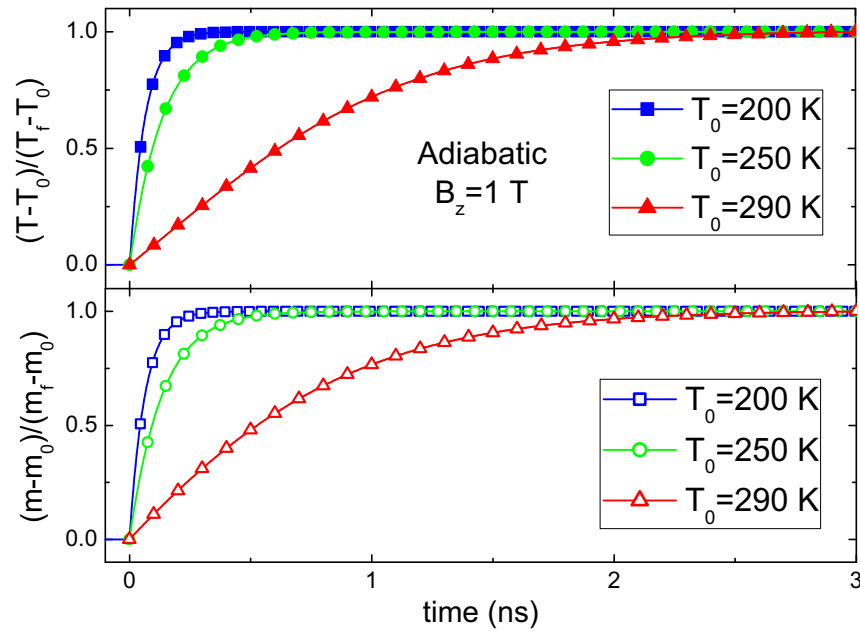

FIG. 3. (Upper panel) Normalized temperature and (lower panel) magnetization dynamics of $\mathrm{Gd}$ induced by the application of an external magnetic field of $B_{z}=\mu_{0} H_{z}=1 \mathrm{~T}$ in adiabatic conditions at three different initial temperatures $T_{0}=200,250$, and $290 \mathrm{~K}$. It was obtained by the integration of the qLLB equation (5) coupled to Eq. (15).

where $m_{h}$ is the field-induced equilibrium magnetization at $T_{c}$. This fact is not a problem in current magnetocaloric refrigeration devices working at temperatures close to $T_{c}$ since their usual cycle frequency is quite slow (of the order of $10 \mathrm{~Hz}$ ) [31]. Typically in this case, the longitudinal relaxation of the system as whole is defined by the presence of interacting grains with the relaxation time via both (small) exchange and dipolar interactions and is much larger than the present case where the exchange interactions are large. However, the present case may be important in future high-frequency devices based on terahertz laser $[34,35]$.

\section{B. Magnetic transverse dynamics}

One of the most promising nanomedical approaches for cancer treatment is magnetic fluid hyperthermia (MFH) [7-9] which takes advantage of the spin-heat dynamics coming from the magnetic transverse relaxation. This technique makes use of alternating external magnetic fields to heat magnetic nanoparticles, previously delivered within the tumor, to locally kill cancer cells (which are more sensitive to a temperature increase $\Delta T$ than the healthy ones).

However, to date only limited clinical-application success has been reached [9]. The main difficulty hampering its further development is the poor knowledge of both (i) the heatdissipation performance of magnetic nanoparticle systems and (ii) of the adequate conditions for efficient heat-triggered cancer treatment. A puzzling example is the MFH-induced cell death with negligible $\Delta T$ (of the whole system) reported in several cases [36,37], which suggests that local heating (i.e., at the particle nanoenvironment level) might be enough to induce the cell apoptosis [38]. Some recent works reported large $\Delta T$ values at the particle nanoenvironment that could justify the local damage within the cells [39,40]. However, those large $\Delta T$ values are in conflict with classical models of 
heat conduction mechanism, largely exceeding the predicted values [41].

In this context, the need to carry out theoretical research able to embrace both the heat generation under the ac field, mediated by the spin-phonon coupling, with the rate of heat release to the environment occurring during the dynamic process is clear.

Typically, the MFH phenomena involve large number of nanoparticles and time scales which range from nanoseconds (magnetization transverse dynamics) to minutes (macroscopic measured temperature of the nanoparticles environment). Consequently, it might be argued that the use of a magnetic equation of motion, as the self-consistent spin-phonon model presented here, may be not suitable in the simplified version for an overall description of magnetic hyperthermia (other techniques as kinetic Monte Carlo modeling are more suitable for the involved time scales) [42]. Note, however, that the specific aspect we aim to undertake here is not the investigation of hyperthermia issues in the usual approach (i.e., good heating capabilities), but we aim, instead, to shed some light on the complex dynamics of the temperature-rise and heat-dissipation counterbalance. In this sense, the present model is likely to provide insightful information.

For simplicity, we considered a single-domain nanoparticle made of magnetite $\left(\mathrm{Fe}_{3} \mathrm{O}_{4}\right)$ described by one single macrospin. We take into account the cubic anisotropy field $\mathbf{H}_{K}=-\left(1 / M_{s}\right)\left(\partial E_{K} / \partial \mathbf{m}\right)$, where $E_{K}=\widetilde{K}_{1}^{(c)}\left[\left(m_{x}^{2} m_{y}^{2}+\right.\right.$ $\left.\left.m_{x}^{2} m_{z}^{2}+m_{y}^{2} m_{z}^{2}\right) / m_{e}^{4}\right]+\widetilde{K}_{2}^{(c)}\left[\left(m_{x}^{2} m_{y}^{2} m_{z}^{2}\right) / m_{e}^{6}\right], \quad \widetilde{K}_{1}^{(c)}$ and $\widetilde{K}_{2}^{(c)}$ are the cubic anisotropy constants, and the external field $\mathbf{H}$, so that $\mathbf{h}=\mathbf{H}_{K}+\mathbf{H}$. The parameters of $\mathrm{Fe}_{3} \mathrm{O}_{4}$ used in the simulations are given by Table I. Moreover, we assume that the nanoparticle is spatially fixed, so that only Néel switching mechanism takes place; such assumption is borne out by experimental works reporting negligible contribution of Brownian rotation to heat dissipation in the cellular environment [43]. We also consider that it interchanges heat with its surroundings through Newton's law of cooling, therefore, we use the qLLB equation (5) coupled to Eq. (18). The initial magnetization is along the easy direction $\langle 1,1,1\rangle$, that is, $\mathbf{m}=\left(m_{e} / \sqrt{3}\right)(1,1,1)$. The size of the nanoparticle in this simple model is related to $\tau_{\text {th }}$ through $\tau_{\text {th }}=\left(V C_{p h}\right) /(A \sigma)$, where $V$ and $A$ are the volume and surface area of the nanoparticle, respectively, $C_{p h}$ is the phonon heat capacity per unit volume, and $\sigma$ is the heat transfer coefficient.

In Fig. 4, we show the numerical results of the magnetization and phonon bath temperature responses to an alternating magnetic field $\mathbf{B}(t)=(0.2 / \sqrt{3})(-1,-1,-1) \sin (2 \pi v t) \mathrm{T}$ (i.e., along the easy-axis direction) with frequency $v=$ $10 \mathrm{MHz}$ for different values of $\tau_{\mathrm{th}}=0.5,5$, and $20 \mathrm{~ns}$. Notice that the magnetization dynamics in the three cases is approximately the same due to the small change in the bath temperature. We see that as $\tau_{\text {th }}$ increases, the time-averaged temperature of the nanoparticle also increases. This is because the heat dissipation rate of the nanoparticle becomes slower and more energy is saved in the phonon bath during the magnetic relaxation. The small reduction of the phonon temperature observed initially is due to the magnetic longitudinal relaxation induced by the external field applied, opposite to the magnetization. Furthermore, note that depending on the

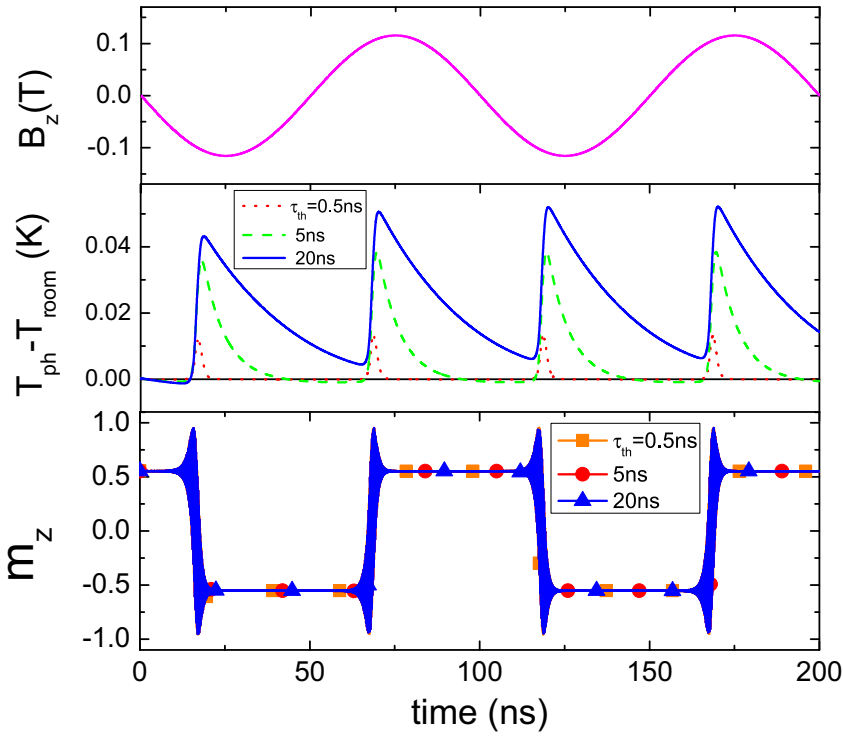

FIG. 4. The $z$ component of the external applied magnetic field (upper panel), phonon bath temperature (middle panel), and $z$ component of the magnetization (lower panel) dynamics in $\mathrm{Fe}_{3} \mathrm{O}_{4}$ calculated using the qLLB equation (5) coupled to Eq. (18) for different values of $\tau_{\text {th }}=0.5,5$, and $20 \mathrm{~ns}$.

frequency of the ac field $v$ and the $\tau_{\text {th }}$ value, the particle may experience a completely different self-heating effect (either due to the environment alone or because of the particles not having enough time to dissipate the energy), and this will in turn influence the heating properties [44]. It is therefore crucial to develop precise methods for accurate estimation of the energy dissipation in order to develop better modeling tools able to guide the experimental advancements in the MFH research area.

\section{CONCLUSIONS}

In summary, we have presented a self-consistent spinphonon dynamical model based on the qLLB equation, which includes not only the action of the thermal bath on magnetization, but also the inverse effect, that is, phonon temperature change induced by magnetization relaxation. Note that a very simple spin-phonon Hamiltonian, where interaction is linear in the spin operator, is used here. However, the model is also directly valid for simple spin-electron Hamiltonian [23] and may be generalized in the future to more complicated situations. The self-consistent spin-phonon dynamical model will be useful to model interesting phenomena where the magnetic and temperature dynamics are relevant, such as HAMR, ultrafast magnetism, magnetic refrigeration, magnetic hyperthermia, or spincaloritronics.

We have provided two simple examples of the use of the model, related to longitudinal and transverse magnetic relaxation, and inspired by magnetic refrigeration and magnetic hyperthermia phenomena. In the first case, we calculated the refrigeration cycle of $\mathrm{Gd}$. The numerical results show that the magnetocaloric effect is higher close to $T_{c}$ in agreement with experiments. In the second case, we have used the model to calculate the magnetization and phonon temperature 
responses of a $\mathrm{Fe}_{3} \mathrm{O}_{4}$ nanoparticle to an alternative external magnetic field. Noteworthy, our results might contribute to understanding the current debate in the literature regarding the existence of large $\Delta T$ local values at nanoparticle surface.

Note that, generally speaking, our model is too simplified to claim an overall correct modeling of the above-mentioned phenomena within our approach, but it opens perspectives to construct more realistic models for their better understanding. Note that we did not consider an important energy-dissipating mechanism, related to excitation of nonhomogeneous magnetization structures as well as we did not take into account longrange spin waves. This can be overcome by considering micromagnetic simulations based on qLLB as in Refs. [18,19,21]. Furthermore, our diffusion model for the temperature to the environment is too simplified and should be substituted in the future by more realistic models [45]. We believe that the model presented in this work constitutes a step forward towards more realistic modeling of spincaloritronic dynamics.

\section{ACKNOWLEDGMENTS}

This work was supported by the Spanish Ministry of Economy and Competitiveness under the Grant No. MAT201347078-C2-2-P. D.S. acknowledges financial support from Xunta de Galicia (Spain) under Plan I2C. P.N. acknowledges support from EU Horizon 2020 Framework Programme for Research and Innovation under Grant Agreement No. 686056, NOVAMAG.

\section{APPENDIX A: GENERAL THEORY FOR A SPIN SYSTEM INTERACTING WEAKLY WITH A THERMAL BATH}

In this Appendix, we summarize the general theory of the spin system interacting weakly with a thermal bath, necessary for the present study [24]. The starting point is the Hamiltonian of the spin system interacting with a bath, given by

$$
\hat{\mathcal{H}}(t)=\hat{\mathcal{H}}_{0}(t)+\hat{V}, \quad \hat{\mathcal{H}}_{0}(t)=\hat{\mathcal{H}}_{s}(t)+\hat{\mathcal{H}}_{b},
$$

where $\hat{\mathcal{H}}_{s}$ and $\hat{\mathcal{H}}_{b}$ are the Hamiltonians of the spin system and the bath, respectively, and $\hat{V}$ describes the interaction between them. Here, we consider that the total Hamiltonian $\hat{\mathcal{H}}(t)$ is time dependent through the nonperturbed Hamiltonian of the spin system $\hat{\mathcal{H}}_{S}(t)$. The interaction operator $\hat{V}$ has the general form

$$
\begin{aligned}
\hat{V}= & \sum_{j} \hat{P}_{j} \hat{F}_{j}, \quad \text { (A2) } \quad \begin{array}{l}
\text { calculated. This procedure leads to } \\
\frac{d}{d t}\left\langle\hat{S}_{i}\right\rangle=
\end{array} \\
& \frac{d}{d t} \operatorname{Tr}_{s}\left\{\hat{\rho}_{s}(t) \hat{S}_{i}\right\}=-\frac{i}{\hbar} \operatorname{Tr}_{s}\left\{\left[\hat{\mathcal{H}}_{s}(t), \hat{\rho}_{s}(t)\right] \hat{S}_{i}\right\}-\frac{i}{\hbar} \operatorname{Tr}_{s}\left(\operatorname{Tr}_{b}\{[\hat{V}, \hat{\rho}(0)]\} \hat{S}_{i}\right) \\
& -\frac{1}{\hbar^{2}} \int_{0}^{t} d t^{\prime} \operatorname{Tr}_{s}\left(\operatorname{Tr}_{b}\left\{\left[\hat{V},\left[\hat{V}\left(t^{\prime}-t\right)_{I}, \hat{U}_{0}^{\dagger}\left(t^{\prime}-t\right) \hat{\rho}\left(t^{\prime}\right) \hat{U}_{0}\left(t^{\prime}-t\right)\right]\right]\right\} \hat{S}_{i}\right), \quad i=x, y, z
\end{aligned}
$$

where $\hat{P}_{j}$ and $\hat{F}_{j}$ are operators acting only on the spin system and bath, respectively.

The time evolution of the density matrix operator $\hat{\rho}(t)$ is governed by the quantum Liouville equation

$$
\frac{d \hat{\rho}(t)}{d t}=-\frac{i}{\hbar}[\hat{\mathcal{H}}(t), \hat{\rho}(t)] .
$$

From this equation, one arrives to [24]

$$
\begin{aligned}
\frac{d}{d t} \hat{\rho}(t)= & -\frac{i}{\hbar}\left[\hat{\mathcal{H}}_{0}(t), \hat{\rho}(t)\right]-\frac{i}{\hbar}[\hat{V}, \hat{\rho}(0)] \\
& -\frac{1}{\hbar^{2}} \int_{0}^{t} d t^{\prime}\left[\hat{V},\left[\hat{V}\left(t^{\prime}-t\right)_{I}, \hat{U}_{0}^{\dagger}\left(t^{\prime}-t\right) \hat{\rho}\left(t^{\prime}\right) \hat{U}_{0}\left(t^{\prime}-t\right)\right]\right]
\end{aligned}
$$

where $\hat{U}_{0}(t)=\hat{U}_{s, 0}(t) \hat{U}_{b, 0}(t)$ is the nonperturbed time evolution operator evaluated at instant $t, \hat{U}_{s, 0}(t)$ and $\hat{U}_{b, 0}(t)=$ $\exp \left(-i \hat{\mathcal{H}}_{b} t / \hbar\right)$ are the nonperturbed time evolution operators of the spin system and bath, respectively, and $\hat{V}(t)_{I}=$ $\hat{U}_{0}^{\dagger}(t) \hat{V} \hat{U}_{0}(t)$ is the interaction operator in the interaction picture.

Now, it is assumed that the interaction between the spin system and the bath is weak, which means that entanglement between them is small, thus, the density operator of the whole system can be factorized. Additionally, it is considered that the bath is at thermal equilibrium (quasiequilibrium), then the density matrix operator can be written as

$$
\hat{\rho}(t) \cong \hat{\rho}_{s}(t) \hat{\rho}_{b}^{e q}(t), \quad \hat{\rho}_{b}^{e q}(t)=\frac{1}{Z_{b}(t)} \exp \left(-\frac{\hat{\mathcal{H}}_{b}}{k_{B} T_{b}(t)}\right),
$$

where $\hat{\rho}_{s}(t)$ and $\hat{\rho}_{b}^{e q}(t)$ are the density matrix operators of the spin system and bath, respectively, $Z_{b}(t)=$ $\operatorname{Tr}_{b}\left(\exp \left\{-\hat{\mathcal{H}}_{b} /\left[k_{B} T_{b}(t)\right]\right\}\right)$ is the bath partition function where $\mathrm{Tr}_{b}$ is the partial trace over the bath variables, and $T_{b}$ is the bath temperature.

The time evolution of the spin operator can be obtained from Eqs. (A4) and (A5) in the following way. First, the partial trace over the bath variable in Eq. (A4) is performed, next it is multiplied by one of the components of the spin operator $\hat{S}_{i}$, $i=x, y, z$, and then the partial trace over the spin variable is

where $\langle\ldots\rangle$ stands for the expectation value of the spin component.

On the other hand, the time evolution of the bath energy can also be obtained from Eqs. (A4) and (A5) by calculating the partial trace over the spin system, multiplying by $\hat{\mathcal{H}}_{b}$ and then performing the partial trace over the bath variable, that is,

$$
\begin{aligned}
\frac{d}{d t}\left\langle\hat{\mathcal{H}}_{b}\right\rangle= & \frac{d}{d t} \operatorname{Tr}_{b}\left\{\hat{\rho}_{b}^{e q}(t) \hat{\mathcal{H}}_{b}\right\}=-\frac{i}{\hbar} \operatorname{Tr}_{b}\left(\operatorname{Tr}_{s}\{[\hat{V}, \hat{\rho}(0)]\} \hat{\mathcal{H}}_{b}\right) \\
& -\frac{1}{\hbar^{2}} \int_{0}^{t} d t^{\prime} \operatorname{Tr}_{b}\left(\operatorname{Tr}_{s}\left\{\left[\hat{V},\left[\hat{V}\left(t^{\prime}-t\right)_{I}, \hat{U}_{0}^{\dagger}\left(t^{\prime}-t\right) \hat{\rho}\left(t^{\prime}\right) \hat{U}_{0}\left(t^{\prime}-t\right)\right]\right]\right\} \hat{\mathcal{H}}_{b}\right) .
\end{aligned}
$$


Therefore, this equation describes the bath energy dynamics due to the interaction only with the spin system. This means that it is valid for adiabatic conditions, where there is no heat transfer with the material environment. Notice that an external magnetic field can induce energy changes in the phonon bath indirectly via the excitation of the spin system.

\section{APPENDIX B: DERIVATION OF THE QLLB EQUATION}

In this Appendix, we present a short summary of the derivation of the qLLB equation [22,23], which may help to understand the approximations, limitations, and future possible improvements of the self-consistent description of spin-phonon dynamics presented in this work.

First, the second term in the right-hand side of Eq. (A6) is zero for the interaction operators considered in the spinphonon model. On the other hand, the analytical calculation of the integrals in Eq. (A6) is complicated. However, with the help of the Markov approximation [24], which is equivalent to the replacement $\hat{U}_{0}^{\dagger}\left(t^{\prime}-t\right) \hat{\rho}\left(t^{\prime}\right) \hat{U}_{0}\left(t^{\prime}-t\right) \rightarrow \hat{\rho}(t)$ in the integrand, and assuming that the retarded spin operator $\hat{\mathbf{S}}\left(t^{\prime}-\right.$ $t)_{I}$ precesses around the field $\mathbf{H}^{\mathrm{MFA}}(t)=H^{\mathrm{MFA}}(t) \mathbf{e}_{z}$, the integrals can be calculated analytically. The approximations are based on the fact that the kernel of the integrals are localized in $\left|t-t^{\prime}\right| \leqslant 1 / w_{\max }$, where $w_{\max }$ is the maximal frequency of the phonon excitations $[22,46]$. In order to avoid unnecessary tedious calculations, the field $\mathbf{H}^{\mathrm{MFA}}$ is considered to be along the $z$ axis, and at the end the general case is deduced from it. As a result, the integrals in Eq. (A6) become [22]

$$
\begin{aligned}
& \int_{0}^{t} d t^{\prime} \boldsymbol{\eta} \cdot \hat{\mathbf{S}}\left(t^{\prime}-t\right)_{I} \sum_{i, j}\left\langle\hat{F}_{i} \hat{F}_{j}\left(t^{\prime}-t\right)\right\rangle \\
& \simeq \eta_{z} \hat{S}_{z} W_{1}+\frac{W_{2}}{2}\left(\eta^{+} \hat{S}^{-}+e^{-y_{0}} \eta^{-} \hat{S}^{+}\right), \\
& \int_{0}^{t} d t^{\prime} \boldsymbol{\eta} \cdot \hat{\mathbf{S}}\left(t^{\prime}-t\right)_{I} \sum_{i, j}\left\langle\hat{F}_{i}\left(t^{\prime}-t\right) \hat{F}_{j}\right\rangle \\
& \simeq \eta_{z} \hat{S}_{z} W_{1}+\frac{W_{2}}{2}\left(e^{-y_{0}} \eta^{+} \hat{S}^{-}+\eta^{-} \hat{S}^{+}\right),
\end{aligned}
$$

where $y_{0}=\beta \hbar \gamma H^{\mathrm{MFA}}, \quad \beta=1 /\left(k_{B} T_{p h}\right), \quad \hat{S}^{ \pm}=\hat{S}_{x} \pm i \hat{S}_{y}$, $\eta^{ \pm}=\eta_{x} \pm i \eta_{y}$, and

$$
\begin{gathered}
W_{1}=\sum_{q, p}\left|V_{p, q}\right|^{2} n_{p}\left(n_{q}+1\right) \pi \delta\left(\omega_{q}-\omega_{p}\right), \\
W_{2}=\sum_{q}\left|V_{q}\right|^{2}\left(n_{q}+1\right) \pi \delta\left(\omega_{q}-\gamma H^{\mathrm{MFA}}\right) \\
+\sum_{p, q}\left|V_{p, q}\right|^{2} n_{p}\left(n_{q}+1\right) \pi \delta\left(\omega_{q}-\omega_{p}-\gamma H^{\mathrm{MFA}}\right),
\end{gathered}
$$

where $n_{q}=\left[\exp \left(\beta \hbar \omega_{q}\right)-1\right]^{-1}$ is the Bose-Einstein distribution. Thus, using Eqs. (B1) and (B2) in Eq. (A6), one finds

$$
\begin{aligned}
\frac{d}{d t}\left\langle\hat{S}_{i}\right\rangle= & -\frac{i \gamma H^{\mathrm{MFA}}(t)}{\hbar}\left\langle\left[\hat{S}_{i}, \hat{S}_{z}\right]\right\rangle-\frac{\eta_{z} W_{1}}{\hbar^{2}}\left\langle\left[\left[\hat{S}_{i}, \boldsymbol{\eta} \cdot \hat{\mathbf{S}}\right], \hat{S}_{z}\right]\right\rangle-\frac{W_{2}}{2 \hbar^{2}}\left\langle\left[\hat{S}_{i}, \boldsymbol{\eta} \cdot \hat{\mathbf{S}}\right]\left(\eta^{+} \hat{S}^{-}+e^{-y_{0}} \eta^{-} \hat{S}^{+}\right)\right\rangle \\
& -\frac{W_{2}}{2 \hbar^{2}}\left\langle\left(e^{-y_{0}} \eta^{+} \hat{S}^{-}+\eta^{-} \hat{S}^{+}\right)\left[\boldsymbol{\eta} \cdot \hat{\mathbf{S}}, \hat{S}_{i}\right]\right\rangle, \quad i=x, y, z .
\end{aligned}
$$

This equation can be simplified with the help of the secular approximation [24], where the nonsecular terms are neglected. It forces the coarse-grained time interval to be [24] $\Delta t \gg$ $\hbar /\left(E_{m}-E_{n}\right)$ where $E_{m(n)}$ is an eigenvalue of $\hat{\mathcal{H}}_{s}$. As a result, Eq. (B5) is simplified to

$$
\begin{array}{r}
\frac{d}{d t}\left\langle\hat{S}_{x(y)}\right\rangle= \\
+\gamma H^{\mathrm{MFA}}\left\langle\hat{S}_{y(x)}\right\rangle-\left(K_{1}+K_{2}\right)\left\langle\hat{S}_{x(y)}\right\rangle \\
+\frac{K_{2}}{\hbar} \tanh \left(\frac{y_{0}}{2}\right)\left\langle\hat{S}_{x(y)} \hat{S}_{z}+\hat{S}_{z} \hat{S}_{x(y)}\right\rangle, \\
\frac{d}{d t}\left\langle\hat{S}_{z}\right\rangle=-2 K_{2}\left\langle\hat{S}_{z}\right\rangle-\frac{2}{\hbar} K_{2} \tanh \left(\frac{y_{0}}{2}\right)\left\langle\hat{S}_{x}^{2}+\hat{S}_{y}^{2}\right\rangle,
\end{array}
$$

where

$$
\begin{gathered}
K_{1}=\eta_{z}^{2} W_{1}, \\
K_{2}=\frac{1}{4}\left(1+e^{-y_{0}}\right)\left(\eta_{x}^{2}+\eta_{y}^{2}\right) W_{2} .
\end{gathered}
$$

Next, the spin operator averages are calculated using the method of the modeling distribution functions [47], assuming a suitable form for the spin density operator as follows;

$$
\hat{\rho}_{s}(t)=\mathcal{Z}_{s}^{-1} \exp \left[-\frac{\mathbf{y}(t) \cdot \hat{\mathbf{S}}}{\hbar}\right], \quad \mathcal{Z}_{s}=\sum_{m=-S}^{S} \exp [-y m],
$$

where $\mathbf{y}(t)$ is an auxiliary dimensionless time-dependent function and its equilibrium value is $\mathbf{y}_{0}=\beta \gamma \hbar \mathbf{H}^{\mathrm{MFA}}=$ $\beta \mu_{a t} \mathbf{H}^{\mathrm{MFA}} / S$. It is possible to show [22] that $\mathbf{y}(t)$ is related to the time-dependent reduced magnetization $\mathbf{m}(t)=$ $-\langle\hat{\mathbf{S}}(t)\rangle / \hbar S$ as

$$
\mathbf{m}(t)=B_{S}[S y(t)] \frac{\mathbf{y}(t)}{y(t)},
$$

where $B_{S}(x)=[(2 S+1) / 2 S] \operatorname{coth}([2 S+1] x / 2 S)-(1 / 2 S)$ $\operatorname{coth}(x / 2 S)$ is the Brillouin function for the spin value $S$. With this method, one finds [22]

$$
\begin{aligned}
\left\langle\hat{S}_{i} \hat{S}_{j}+\hat{S}_{j} \hat{S}_{i}\right\rangle= & \frac{S m \hbar^{2} \delta_{i j}}{\tanh \left(\frac{y}{2}\right)}+\frac{S m \hbar^{2}}{\tanh \left(\frac{y}{2}\right)} \\
& \times\left[\frac{2(S+1) \tanh \left(\frac{y}{2}\right)}{m}-3\right] \frac{m_{i} m_{j}}{m^{2}} .
\end{aligned}
$$


Hence, using this equation in Eqs. (B6) and (B7) and considering the general case where $\mathbf{H}^{\mathrm{MFA}}$ is not necessary parallel to the $z$ axis, one arrives to Eq. (4) [22].

\section{APPENDIX C: SPIN-PHONON ENERGY CONSERVATION}

In this Appendix, we prove the energy conservation between spins and phonons in our model through the first law of thermodynamics. For simplicity and following the original qLLB equation derivation in Ref. [22], here we consider the two-ion (exchange) anisotropy, that is, the MFA field of each magnetic ion is given by

$\mathbf{H}_{i}^{\mathrm{MFA}}(t)=\frac{J_{0}}{\mu_{a t}}\left[\mathbf{m}_{i}(t)-\tilde{\eta}_{x} m_{x, i}(t) \mathbf{e}_{x}-\tilde{\eta}_{y} m_{y, i}(t) \mathbf{e}_{y}\right]+\mathbf{H}(t)$,

where $\tilde{\eta}_{x(y)}$ are small dimensionless exchange anisotropy constants with $\left|\widetilde{\eta}_{x(y)}\right| \ll 1$. Next, from the first law of thermodynamics we find that the time derivative of the total energy (spins and bath) in adiabatic conditions is given by the work per unit time done by the external field on the spin system, that is,

$$
\frac{d\left\langle\hat{\mathcal{H}}_{\mathrm{tot}}\right\rangle}{d t}=\frac{d\left\langle\hat{\mathcal{H}}_{b}\right\rangle^{\mathrm{tot}}}{d t}+\frac{d\left\langle\hat{\mathcal{H}}_{s}^{\mathrm{tot}}\right\rangle}{d t}=-\mu_{a t} \sum_{i=1}^{N} \mathbf{m}_{i} \cdot \frac{d \mathbf{H}}{d t}
$$

where $N$ is the total number of spins,

$$
\frac{d\left\langle\hat{\mathcal{H}}_{b}\right\rangle^{\text {tot }}}{d t}=\sum_{i=1}^{N} \frac{d\left\langle\hat{\mathcal{H}}_{b}\right\rangle^{i}}{d t}
$$

is the time derivative of total bath energy expectation value, and $\left\langle\hat{\mathcal{H}}_{s}^{\text {tot }}\right\rangle$ is the total energy expectation value of the spin system, that is,

$$
\left\langle\hat{\mathcal{H}}_{s}^{\mathrm{tot}}\right\rangle(t)=-\mu_{a t} \sum_{i=1}^{N} \widetilde{\mathbf{H}}_{i}^{\mathrm{MFA}}(t) \cdot \mathbf{m}_{i}(t),
$$

where

$\widetilde{\mathbf{H}}_{i}^{\mathrm{MFA}}(t)=\frac{J_{0}}{2 \mu_{a t}}\left[\mathbf{m}_{i}(t)-\tilde{\eta}_{x} m_{x, i}(t) \mathbf{e}_{x}-\tilde{\eta}_{y} m_{y, i}(t) \mathbf{e}_{y}\right]+\mathbf{H}(t)$.

Notice that $\widetilde{\mathbf{H}}_{i}^{\mathrm{MFA}}$ is different to $\mathbf{H}^{\mathrm{MFA}}$ [see Eq. (C1)] because it contains an extra factor $\frac{1}{2}$ in the exchange field due to the spin double counting in the summation done in Eq. (C4). Next, we derive Eq. (C4) with respect to time

$$
\frac{d\left\langle\hat{\mathcal{H}}_{s}^{\mathrm{tot}}\right\rangle}{d t}=-\mu_{a t} \sum_{i=1}^{N} \mathbf{H}_{i}^{\mathrm{MFA}} \cdot \frac{d \mathbf{m}_{i}}{d t}-\mu_{a t} \sum_{i=1}^{N} \mathbf{m}_{i} \cdot \frac{d \mathbf{H}}{d t} .
$$

Finally, from Eqs. (C2), (C3), and (C6) we find

$$
\frac{d\left\langle\hat{\mathcal{H}}_{b}\right\rangle^{i}}{d t}=\mu_{a t} \mathbf{H}_{i}^{\mathrm{MFA}} \cdot \frac{d \mathbf{m}_{i}}{d t}, \quad i=1, \ldots, N
$$

which is the same time derivative of the bath energy per spin than in Eq. (13).

\section{APPENDIX D: ELECTRON-IMPURITY MODEL}

In this Appendix, we derive a self-consistent description of the spin-electron bath dynamics using the electron-impurity scattering model proposed by Koopmans et al. in Ref. [48] and Dalla Longa in Ref. [49] for the laser-induced magnetization dynamics. The Hamiltonian consists of a spin system which weakly interacts with a spinless electron bath and it reads as

$$
\hat{\mathcal{H}}=\hat{\mathcal{H}}_{s}+\hat{\mathcal{H}}_{e}+\hat{V}_{s-e},
$$

where $\hat{\mathcal{H}}_{s}$ is the energy of the spin system, $\hat{\mathcal{H}}_{e}$ stands for the electron bath energy, and $\hat{V}_{s-e}$ describes the spin-electron interaction energy:

$$
\begin{gathered}
\hat{\mathcal{H}}_{s}=\gamma \mathbf{H}^{\mathrm{MFA}} \cdot \hat{\mathbf{S}}, \\
\hat{\mathcal{H}}_{e}=\sum_{\mathbf{k}}\left(\epsilon_{\mathbf{k}}-\mu\right) \hat{c}_{\mathbf{k}}^{\dagger} \hat{c}_{\mathbf{k}}, \\
\hat{V}_{s-e}=\sum_{\mathbf{k}, \mathbf{k}^{\prime}} V_{\mathbf{k}, \mathbf{k}^{\prime}}\left(\hat{S}^{+}+\hat{S}^{-}\right) \hat{c}_{\mathbf{k}}^{\dagger} \hat{c}_{\mathbf{k}^{\prime}} .
\end{gathered}
$$

Here, $\hat{c}_{\mathbf{k}}^{\dagger}\left(\hat{c}_{\mathbf{k}}\right)$ is the creation (annihilation) operator which creates (annihilates) an electron with momentum $\mathbf{k}, \epsilon_{\mathbf{k}}=$ $\hbar^{2} k^{2} /\left(2 m_{e l}\right), m_{e l}$ is the electron mass, $\mu$ is the chemical potential, $V_{\mathbf{k}, \mathbf{k}^{\prime}}$ describes the scattering amplitude. The vector $\mathbf{H}^{\mathrm{MFA}}$ is given by Eq. (3).

In our previous work [23], we already showed that the qLLB equation can be obtained for this model. In particular, we found the same qLLB equation given by Eq. (5) where now the temperature corresponds to the electron bath temperature $T_{e}, K_{1}=0, K_{2}=\left(1+e^{-y_{0}}\right) W_{2}$ with $y_{0}=\beta \hbar \gamma H^{\mathrm{MFA}}, \beta=$ $1 /\left(k_{B} T_{e}\right)$, and

$$
W_{2}=2 \pi \sum_{\mathbf{k}, \mathbf{k}^{\prime}}\left|V_{\mathbf{k}, \mathbf{k}^{\prime}}\right|^{2} \widetilde{n}_{\mathbf{k}^{\prime}}\left(1-\tilde{n}_{\mathbf{k}}\right) \delta\left(\gamma H^{\mathrm{MFA}}-\frac{\epsilon_{\mathbf{k}}-\epsilon_{\mathbf{k}^{\prime}}}{\hbar}\right),
$$

where $\tilde{n}_{\mathbf{k}}=\left[\exp \left(\beta\left(\epsilon_{\mathbf{k}}-\mu\right)\right)+1\right]^{-1}$ is the Fermi-Dirac distribution. Moreover, the damping parameters are given by

$$
\begin{gathered}
\alpha_{\|}= \begin{cases}\lambda \frac{2 T_{e}}{3 T_{c}} \frac{2 q_{s}}{\sinh \left(2 q_{s}\right)}, & T_{e} \lesssim T_{c} \\
\lambda \frac{2 T_{e}}{3 T_{c}}, & T_{e}>T_{c}\end{cases} \\
\alpha_{\perp}= \begin{cases}\lambda\left[\frac{\tanh \left(q_{s}\right)}{q_{s}}-\frac{2 T_{e}}{3 T_{c}}\right], & T_{e} \lesssim T_{c} \\
\lambda \frac{T_{e}}{3 T_{c}}, & T_{e}>T_{c}\end{cases}
\end{gathered}
$$

where $\epsilon=\left(T_{c}-T_{e}\right) / T_{c}, q_{s}=3 T_{c} m_{e} /\left[2(S+1) T_{e}\right]$, and

$$
\lambda=K_{2} \frac{(S+1)}{S} \frac{\mu_{a t}}{\gamma k_{B} T_{e}} .
$$

Following the same procedure as in spin-phonon interaction model described in Sec. II C, we find that for the electronimpurity model the time derivative of the electron bath temperature induced by the spin dynamics is

$$
\frac{d T_{e}}{d t}=\frac{\gamma \alpha_{\|} M_{s} J_{0}}{C_{e} \mu_{a t}} \mathbf{m} \cdot \mathbf{H}_{\mathrm{eff}}+\frac{\gamma \alpha_{\perp} M_{s}}{C_{e}} \frac{(\mathbf{m} \times \mathbf{h})^{2}}{m^{2}} \equiv f\left(\mathbf{m}, T_{e}\right),
$$

where $C_{e}$ is the electron bath specific heat in units of $\mathrm{JK}^{-1} \mathrm{~m}^{-3}$ and $\mathbf{H}_{\mathrm{eff}}$ is given by Eq. (6) with bath temperature equals to $T_{e}$. 
[1] M. H. Kryder, E. C. Gage, T. W. McDaniel, W. A. Challener, R. E. Rottmayer, J. Ganping, H. Yiao-Tee, and M. F. Erden, Proc. IEEE 96, 1810 (2008).

[2] I. L. Prejbeanu, M. Kerekes, R. C. Sousa, H. Sibuet, O. Redon, B. Dieny, and J. P. Nozières, J. Phys.: Condens. Matter 19, 165218 (2007).

[3] E. Beaurepaire, J.-C. Merle, A. Daunois, and J. Y. Bigot, Phys. Rev. Lett. 76, 4250 (1996).

[4] T. A. Ostler, J. Barker, R. F. L. Evans, R. W. Chantrell, U. Atxitia, O. Chubykalo-Fesenko, S. El Moussaoui, L. Le Guyader, E. Mengotti, L. J. Heyderman, F. Nolting, A. Tsukamoto, A. Itoh, D. Afanasiev, B. A. Ivanov, A. M. Kalashnikova, K. Vahaplar, J. Mentink, A. Kirilyuk, Th. Rasing, and A. V. Kimel, Nat. Commun. 3, 666 (2012).

[5] K. Uchida, S. Takahashi, K. Harii, J. Ieda, W. Koshibae, K. Ando, S. Maekawa, and E. Saitoh, Nature (London) 455, 778 (2008).

[6] P. Weiss and A. Piccard, J. Phys. Theor. Appl. 7, 103 (1917).

[7] A. B. Salunkhe, V. M. Khot, and S. H. Pawar, Curr. Top. Med. Chem. 14, 572 (2014).

[8] M. Colombo, S. Carregal-Romero, M. F. Casula, L. Gutiérrez, M. P. Morales, I. B. Böhm, J. T. Heverhagen, D. Prosperi, and W. J. Parak, Chem. Soc. Rev. 41, 4306 (2012).

[9] E. A. Périgo, G. Hemery, O. Sandre, D. Ortega, E. Garaio, F. Plazaola, and F. J. Teran, Phys. Rev. Appl. 2, 041302 (2015).

[10] M. I. Kaganov, I. M. Lifshitz, and L. V. Tanatarov, Zh. Eksp. Teor. Fiz. 31, 232 (1957) [Sov. Phys. JETP 4, 173 (1957)].

[11] Pui-Wai Ma, S. L. Dudarev, and C. H. Woo, Phys. Rev. B 85, 184301 (2012).

[12] M. Dvornik, A. Vansteenkiste, and B. Van Waeyenberge, Appl. Phys. Lett. 105, 162411 (2014).

[13] D. A. Garanin, Phys. Rev. B 55, 3050 (1997).

[14] T. McDaniel, J. Appl. Phys. 112, 013914 (2012).

[15] D. Hinzke and U. Nowak, Phys. Rev. Lett. 107, 027205 (2011).

[16] U. Atxitia, O. Chubykalo-Fesenko, J. Walowski, A. Mann, and M. Münzenberg, Phys. Rev. B 81, 174401 (2010).

[17] M. Sultan, U. Atxitia, A. Melnikov, O. Chubykalo-Fesenko, and U. Bovensiepen, Phys. Rev. B 85, 184407 (2012).

[18] N. Kazantseva, D. Hinzke, U. Nowak, R. W. Chantrell, U. Atxitia, and O. Chubykalo-Fesenko, Phys. Rev. B 77, 184428 (2008).

[19] U. Atxitia, O. Chubykalo-Fesenko, N. Kazantseva, D. Hinzke, U. Nowak, and R. W. Chantrell, Appl. Phys. Lett. 91, 232507 (2007).

[20] K. Vahaplar, A. M. Kalashnikova, A. V. Kimel, D. Hinzke, U. Nowak, R. Chantrell, A. Tsukamoto, A. Itoh, A. Kirilyuk, and Th. Rasing, Phys. Rev. Lett. 103, 117201 (2009).

[21] J. Mendil, P. Nieves, O. Chubykalo-Fesenko, J. Walowski, T. Santos, S. Pisana, and M. Münzenberg, Sci. Rep. 4, 3980 (2014).

[22] D. A. Garanin, Physica A (Amsterdam) 172, 470 (1991).

[23] P. Nieves, D. Serantes, U. Atxitia, and O. Chubykalo-Fesenko, Phys. Rev. B 90, 104428 (2014).
[24] K. Blum, Density Matrix Theory and Applications (Plenum, New York, 1981).

[25] P. Nieves Cordones, Ph.D. thesis, Instituto de Ciencia de Materiales de Madrid (ICMM)-Universidad Autónoma de Madrid, 2015.

[26] O. Chubykalo-Fesenko, U. Nowak, R. W. Chantrell, and D. Garanin, Phys. Rev. B 74, 094436 (2006).

[27] S. M. Bhagat and P. Lubitz, Phys. Rev. B 10, 179 (1974).

[28] Yi Li, K. Baberschke, and M. Farle, J. Appl. Phys. 69, 4992 (1991).

[29] U. Atxitia and O. Chubykalo-Fesenko, Phys. Rev. B 84, 144414 (2011).

[30] K. A. Gschneidner, Jr. and V. K. Pecharsky, Int. J. Refrig. 31, 945 (2008).

[31] A. Kitanovski, J. Tušek, U. Tomc, U. Plaznik, M. Ožbolt, and A. Poredoš, Magnetocaloric Energy Conversion (Springer, Cham, Switzerland, 2015).

[32] A. M. Tishin and Y. I. Spichkin, The Magneto Caloric Effect and Its Applications (Institute of Physics Publishing, Bristol, 2003).

[33] V. K. Pecharsky and K. A. Gschneidner, Jr., J. Magn. Magn. Mater. 200, 44 (1999).

[34] Pui-Wai Ma and S. L. Dudarev, Phys. Rev. B 90, 024425 (2014).

[35] C. Vicario, C. Ruchert, F. Ardana-Lamas, P. M. Derlet, B. Tudu, J. Luning, and C. P. Hauri, Nat. Photonics 7, 720 (2013).

[36] M. Creixell, A. C. Bohorquez, M. Torres-Lugo, and C. Rinaldi, ACS Nano 5, 7124 (2011).

[37] A. Villanueva, P. De La Presa, J. M. Alonso, T. Rueda, A. Martinez, P. Crespo, M. P. Morales, M. A. Gonzalez-Fernandez, J. Valdes, and G. Rivero, J. Phys. Chem. C 114, 1976 (2010).

[38] C. Munoz-Menendez, I. Conde-Leboran, D. Baldomir, O Chubykalo-Fesenko, and D. Serantes, Phys. Chem. Chem. Phys. 17, 27812 (2015).

[39] A. Riedinger, P. Guardia, A. Curcio, M. A. Garcia, R. Cingolani, L. Manna, and T. Pellegrino, Nano Lett. 13, 2399 (2013).

[40] J. T. Dias, M. Moros, P. del Pino, S. Rivera, V Grazú, J. M. de la Fuente, and Jesus M. Angew, Chem. Int. Edit. 52, 11526 (2013).

[41] S. Dutz and R. Hergt, Nanotechnology 25, 452001 (2014).

[42] S. Ruta, R. Chantrell, and O. Hovorka, Sci. Rep. 5, 9090 (2015).

[43] R. Di Corato, A. Espinosa, L. Lartigue, M. Tharaud, S. Chat, T. Pellegrino, C. Ménager, F. Gazeau, and C. Wilhelm, Biomaterials 35, 6400 (2014).

[44] E. Garaio, O. Sandre, J.-M. Collantes, J. A. Garcia, S. Mornet, and F. Plazaola, Nanotechnology 26, 015704 (2015).

[45] H. H. Pennes, J. Appl. Physiol. 1, 93 (1948).

[46] D. A. Garanin, Adv. Chem. Phys. 147, 213 (2012).

[47] D. A. Garanin, V. V. Ishchenko, and L. V. Panina, Teor. Mat. Fiz. 82, 242 (1990) [Theor. Math. Phys. 82, 169 (1990)].

[48] B. Koopmans, J. J. M. Ruigrok, F. Dalla Longa, and W. J. M. de Jonge, Phys. Rev. Lett. 95, 267207 (2005).

[49] F. Dalla Longa, Ph.D. thesis, Eindhoven University of Technology, Eindhoven, The Netherlands, 2008. 